\title{
A CULTURA E O AMBIENTE DE INOVAÇÃO: ESTUDO EM UMA MULTINACIONAL VAREJISTA
}

Andressa Gonçalves ${ }^{1}$ Luciano de Castro Carvalho ${ }^{2}$

Silvana Vieira Tambosi ${ }^{3}$ Denise Del Pra Netto Machado ${ }^{4}$

\section{Resumo}

O objetivo deste estudo foi analisar a influência da cultura organizacional sobre o ambiente de inovação em uma empresa multinacional varejista. Para tal, aplicou-se o questionário adaptado de Hofstede (1980) e de Bates et al. (1995), referente à cultura, e o de Machado, Carvalho e Heinzmann (2012) sobre o ambiente de inovação. A amostra foi de 156 respondentes. Os dados foram analisados por meio de modelagem de equações estruturais. Os resultados indicaram que houve relação positiva na influência da cultura organizacional sobre o ambiente de inovação. Para pesquisas futuras, indica-se ampliar a amostra para outras empresas e estabelecer uma relação entre inovação e cultura.

Palavras-chave: Cultura organizacional. Ambiente de Inovação. Organização. Resultados.

$* * *$

1 Mestre, e-mail: dessa. cool@gmail.com

2 Doutor, e-mail: luccar@ gmail.com

3 Mestre, e-mail: profa. silvana.vieira@gmail. com

4 Doutora, e-mail: profadenisedellpra@ gmail.com 


\section{INTRODUÇÃO}

As mudanças ocorridas no ambiente afetam a gestão das organizações e a forma como elas se posicionam diante das demandas de um mercado cada vez mais dinâmicas, em que há a intensificação da concorrência, mudanças no ambiente tecnológico, econômico e social, tornando o mercado cada vez mais competitivo e turbulento. A crescente complexidade e o ritmo da mudança tecnológica fazem com que as empresas constituam novas alianças com agentes externos e busquem maior flexibilidade e eficiência em seus processos internos, como resposta às mudanças do mercado.

Machado, Carvalho e Heinzmann (2012) consideram que os aspectos internos à organização são os principais propulsores para o aparecimento de novas tecnologias que resultem em inovação. Os autores entendem que a avaliação interna, com o objetivo de indicar as forças e fraquezas, mostra-se como sendo relevante para as empresas. Dentre os aspectos a serem observados nesse ambiente, constatam-se as dimensões relativas aos processos, recursos, liderança, proatividade, relacionamentos e formalização das comunicações.

Não obstante de toda literatura presente acerca da temática da inovação, argumentando a necessidade iminente de mudanças, se uma empresa não possuir uma cultura propícia ao desenvolvimento de inovações, ela poderá chegarao insucesso (MACHADO; CARVALHO; HEINZMANN,2012). Desta forma, é necessário um ambiente que viabilize o desenvolvimento de inovação, composto por pessoas, equipamentos, conhecimentos, recursos financeiros. Além disso, o espaço é afetado também pelas competências organizacionais que irão gerenciar esses recursos, bem como a existência de uma cultura organizacional que estimule o surgimento de novas ideias, a liberdade de expor opiniões e o envolvimento dos empregados com o propósito de gerar e disseminar inovações (TIDD et al., 1997; AHMED, 1998).

\section{A INOVAÇÃO PODE SER VISTA} COMO UM MEIO PELO QUAL

AS EMPRESAS SE DESTACAM

OU SE MANTÉM COMPETITIVAS FRENTE A NOVAS EXIGÊNCIAS MOLDADAS PELO AMBIENTE DE INCERTEZA.

Pérez-Bustamante (1999) ressalta que as organizações estão percebendo a inovação como um dos meios para o alcance dessa competitividade e de sua sobrevivência no mercado. Para o atingimento desse objetivo, é necessário analisar os elementos que compõem o ambiente da organização e seus efeitos sobre o desenvolvimento de inovações.

Sob este contexto, torna-se pertinente o seguinte questionamento: Quais são as implicações da cultura organizacional sobre o ambiente de inovação e o desempenho inovador?

Buscando responder a esta pergunta, a presente pesquisa tem como objetivo analisar a influência da cultura organizacional sobre o ambiente de inovação no contexto de uma empresa multinacional varejista que atua no Brasil.

O presente trabalho está dividido em quatro seções, além desta introdução. No segundo 
segmento, será apresentado o quadro teórico e de hipóteses acerca do ambiente de inovação e da cultura organizacional. $\mathrm{Na}$ terceira seção, serão delineados os procedimentos metodológicos e, por fim, a apresentação e a análise dos resultados da pesquisa.

\section{QUADRO TEÓRICO E HIPÓTESES}

Nesta seção, serão apresentados os conceitos de cultura e ambiente de inovação que serviram de norteadores para a presente pesquisa e, na discussão dos dados, servirão como suporte teórico.

\subsection{Cultura}

A cultura de uma organização consiste em um conjunto de práticas, símbolos, valores, artefatos e pressupostos que as organizações compartilham com relação ao comportamento apropriado, instituído pelos fundadores e criadores da empresa. Os artefatos podem incluir o layout físico, as normas e os regulamentos, a maneira como as pessoas interagem, os aspectos mais permanentes, como documentos, produtos, declarações e relatórios (SCHEIN, 1990).

\section{O CONCEITO DE CULTURA}

ORGANIZACIONAL

FOI APROPRIADO DA

ANTROPOLOGIA, CIÊNCIA NA

QUAL NÃO HÁ UM CONSENSO

SOBRE O SEU SIGNIfICADO MAIS

AMPLO.

Apesar das diversas definições de cultura, quando ela se reporta ao meio organizacional, busca-se uma convergência relativa aos padrões, crenças e valores que se manifestam nas práticas sociais organizacionais, chamadas também de práticas organizacionais (HOFSTEDE et al., 1990).

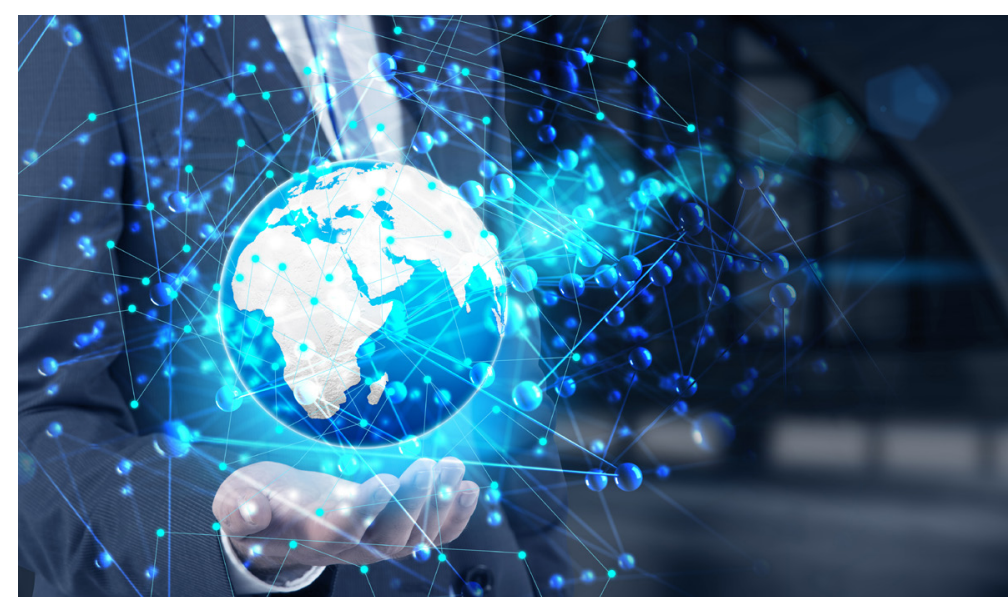

Hofstede et al. (1990) argumentam que vários aspectos da configuração organizacional, como a estrutura, as expectativas dos participantes de uma organização, a forma de se tomar decisões e a consequente resolução de problemas, são influenciadas pela cultura organizacional. Nesta mesma perspectiva,Whittington (2006), apropriando-se de conceitos de campo social de Bourdieu (1989) e da teoria dos sistemas sociais de Giddens (1984), enquadra as práticas em um entendimento que, compartilhadas pelas organizações, se reproduzem por meio de regras culturais, linguagens e procedimentos que irão guiar o comportamento deste grupo. Este contexto varia de acordo com cada organização. Sendo assim, a cultura pode influenciar a forma de como as pessoas se comunicam e compartilham conhecimento em todos os níveis da organização (PETTIGREW, 1982). 
Concatenando nessa mesma linha, Schein (1984) enfatiza que a cultura organizacional é o padrão de pressupostos básicos que um determinado grupo criou. Normalmente tem início com os fundadores da organização, que compartilham, desenvolvem suposições sobre o mundo e ensinam esses pressupostos para toda organização. Este processo de socialização tem por objetivo desenvolver ideais que possibilitam a organização a aprender a lidar com seus problemas de adaptação ao ambiente externo e habilitar a coesão do grupo (BERGER; LUCKMAN, 1967).

Várias tipologias de cultura já foram apresentadas na literatura (SCHNEIDER, 1996; HANDY,1978; QUINN;McGRATH,1985; TROMPENAARS, 1994; HOFSTEDE, 1991). Nesta pesquisa, foram adotados os estudos de Bates et al. (1995), elaboradas incialmente por Georgopoulos e Mann (1962), e Taylor e Bowers (1972). Os autores fazem uma abordagem da congruência cultural, que é medida pela escala da lealdade e da filosofia, além de indicar a credibilidade nas crenças e o reconhecimento do indivíduo nos princípios, normas e regras das organizações.

\section{PARA MENSURAR A CULTURA, BATES ET AL. (1995) \\ EMPREGAM, ALÉM DAS ESCALAS DE LEALDADE E DE filosofiA, DUAS OUTRAS DIMENSÕES, QUE SÃO: (1) DISTÂNCIA DO PODER, OU DISTÂNCIA HIERÁRQUICA, E (2) INDIVIDUALISMO VERSUS COLETIVISMO.}

É importante ressaltar que essas dimensões já foram utilizadas em vários outros estudos, como de Kanungo, Sadavarti e Srinivas (2001); Balthazard e Cooke (2004); e no Brasil, por Machado, Heinzmann e Loesch (2009);
Machado, Carvalho e Heinzmann (2012), Scarpin e Machado (2012).

A dimensão individualismo vesus coletivismo é a medida do quanto os membros de um determinado grupo são responsáveis pelos que estão à sua volta e suportam a interferência do grupo no direcionamento das atividades. Quanto mais individualista, menos laços são formados no grupo. $\mathrm{O}$ coletivismo, por sua vez, preza a prevalência do interesse grupal em detrimento do individual.

Quanto à dimensão distância do poder, ou distância hierárquica, indica a forma como os grupos lidam com as desigualdades entre os indivíduos, referindo-se à distribuição de autoridade. Na grande distância hierárquica, superiores e subordinados se consideram desiguais entre si e quem tem menos poder depende de quem tem mais (HOFSTEDE, 1980).

\subsection{Ambiente de Inovação e Cultura}

A crescente importância do conhecimento na sociedade contemporânea exige uma mudança na maneira de pensar e agir, principalmente no que tange à inovação nas organizações, seja ela de caráter tecnológico, de produto ou processo, de inovação estratégica ou organizacional (ISIDRO FILHO; GUIMARÃES, 2010).

O estudo sobre o processo de inovação das organizações tem evoluído como um esforço multidisciplinar ao longo dos anos, tendo em vista que a capacidade de desenvolver novos produtos e serviços viáveis é de interesse para a maioria das organizações. A inovação permite às organizações melhorarem a qualidade de sua produção, entrar em novos mercados, reagir ao avanço da concorrência e alavancar 
investimentos em novas tecnologias (ETTLIE et al., 1984).

Um dos precursores no estudo da inovação foi Schumpeter (1985). O autor definiu a inovação como sendo as novas combinações de recursos e como o resultado de processos de aprendizagem em que conhecimentos relevantes são combinados e estruturados em novas soluções e significados compartilhados. Neste sentido, a organização inovadora é a que vai oferecer produtos ou que presta serviços com valores agregados, utilizando um novo conhecimento para o seu desenvolvimento e que atendam às necessidades dos consumidores (DAMANPOUR, 1996; AFUAH, 1999).

A capacidade de inovar está diretamente relacionada à cultura da organização, ao conhecimento gerado pelos seus colaboradores, às ideias que surgem, bem como aos processos internos eà adequação às mudanças provenientes do ambiente, com o objetivo de manter ou tornar a organização competitiva no mercado. Identificar qual ambiente é mais ou menos inovador acarreta uma análise em perspectivas distintas de diversos estudiosos da temática, que buscam identificar quais elementos favorecem a um ambiente de inovação (JAMBULINGAM et al.,2005; AKMAN; YILMAZ,2008). Com base na argumentação, delineiam-se as seguintes hipóteses de pesquisa: $\mathbf{H}_{\mathbf{1}}=$ A cultura influencia positivamente o ambiente interno do grupo de inovação; e $\mathbf{H}_{2}=$ A cultura influencia positivamente o ambiente externo ao grupo de inovação.

A inovação não é um trabalho a ser executado por uma só pessoa ou por um departamento específico da organização, mas sim poruma rede de pessoas que cooperam e sejam comprometidas, para que se desenvolvam ideias por meio dessas interações. Assim, a partir da aceitação desses conceitos, consegue-se sua legitimidade (VAN DE VEN, 1989). A partir disso, os pesquisadores do Minnesota Innovation Research Program (MIRP) desenvolveram dimensões para a explicação do processo de inovação, a fim de criar um modelo capaz de identificar o ambiente organizacional favorável ao desenvolvimento de inovações. Esse processo é composto por cinco elementos: ideias, pessoas, transações, contexto e resultados.

No Brasil, esses estudos foram replicados por Machado (2004), Vicenti (2006), Barzotto (2008) e Carvalho (2010), culminando em uma nova metodologia proposta por Machado e Carvalho (2011) para configurar um ambiente propício à inovação, conforme descrito no Quadro 1, a seguir.

Quadro 1: Ambiente de inovação

\begin{tabular}{|c|c|c|l|}
\hline GRUPO & \multicolumn{2}{|c|}{ DIMENSÃO } & DESCRIÇÃO \\
\hline Resultados & D1 & Resultados & $\begin{array}{l}\text { Grau com que as pessoas percebem que a inovação } \\
\text { atendeu às expectativas individuais e da empresa } \\
\text { em termos de processo e resultados. Quanto maior a } \\
\text { percepção da efetividade da inovação, maior a percepção } \\
\text { de um ambiente inovador. } \\
\text { AUTORES: Bates e Evanisko (1981); Van de Ven (1986). }\end{array}$ \\
\hline
\end{tabular}




\begin{tabular}{|c|c|c|c|}
\hline GRUPO & \multicolumn{2}{|c|}{ DIMENSÃO } & DESCRIÇÃO \\
\hline \multirow{6}{*}{$\begin{array}{c}\text { Dimensões } \\
\text { internas ao } \\
\text { grupo de } \\
\text { inovação }\end{array}$} & D2 & Processos & $\begin{array}{l}\text { Refere-se à facilidade de entendimento dos processos } \\
\text { necessários ao desenvolvimento da inovação, } \\
\text { compreendendo regras definidas e procedimentos } \\
\text { padronizados. Quanto mais compreensível for o } \\
\text { processo, maisfavorável para osurgimentodeinovações. } \\
\text { AUTORES: Perrow (1967); Gross et al. (1971); Bass } \\
\text { (1971); Van de Ven e Ferry (1980). }\end{array}$ \\
\hline & D3 & Recursos & $\begin{array}{l}\text { Refere-se à quantidade de recursos disponíveis para o } \\
\text { desenvolvimento de inovações. Níveis moderados de } \\
\text { recursos estão relacionados ao sucesso da inovação. } \\
\text { AUTORES: Lawrence e Dyer (1983). }\end{array}$ \\
\hline & D4 & Liderança & $\begin{array}{l}\text { A percepção de incentivo do comportamento inovador } \\
\text { e de um ambiente livre de punições quando uma ideia } \\
\text { falha estimula os indivíduos a gerarem novas ideias } \\
\text { e aprenderem com os próprios erros. Quanto maior a } \\
\text { percepção da dimensão Liderança, maior sucesso no } \\
\text { resultado da inovação. } \\
\text { AUTORES: Hackman e Oldham (1975); Filley et al. } \\
\text { (1976); Van de Ven e Ferry (1980); Schein (1984). }\end{array}$ \\
\hline & D5 & Autonomia & $\begin{array}{l}\text { Os participantes sentem-se parte do processo e, com } \\
\text { isso, expõem suas ideias e opiniões no intuito de ajudar } \\
\text { no processo decisório referente à inovação. Quanto } \\
\text { mais envolvidos os participantes estiverem no processo, } \\
\text { maiores as chances de obterem sucesso na inovação. } \\
\text { AUTORES: Pressman e Wildavsky (1973); Shull et al. } \\
\text { (1970); Van de Ven (1980); McGrath (1984). }\end{array}$ \\
\hline & D6 & $\begin{array}{l}\text { Relacionamento } \\
\text { interno do } \\
\text { grupo de } \\
\text { inovação }\end{array}$ & $\begin{array}{l}\text { Refere-se ao grau de interação e consenso e forma } \\
\text { de resolução de conflitos entre o grupo de inovação. } \\
\text { Quanto maior a sinergia do grupo de inovação, maior } \\
\text { a probabilidade de sucesso na inovação. } \\
\text { AUTORES: Kanter (1983); Lawrence e Lorsch (1967); } \\
\text { Burke (1970); Van de Ven; Ferry (1980). }\end{array}$ \\
\hline & D7 & $\begin{array}{l}\text { Relacionamento } \\
\text { externo ao } \\
\text { grupo de } \\
\text { inovação }\end{array}$ & $\begin{array}{l}\text { Refere-se ao grau de interação do grupo de inovação } \\
\text { com outros grupos dentro da empresa a fim de buscar } \\
\text { auxílio para o desenvolvimento de inovações e benefícios } \\
\text { mútuos. } \\
\text { AUTORES: Kanter (1983); Blake e Mouton (1964); } \\
\text { Lawrence e Lorsch (1967); Burke (1970); Van de Ven e } \\
\text { Ferry (1980). }\end{array}$ \\
\hline
\end{tabular}




\begin{tabular}{|c|c|c|c|}
\hline GRUPO & \multicolumn{2}{|c|}{ DIMENSÃO } & DESCRIÇÃO \\
\hline \multirow{4}{*}{$\begin{array}{l}\text { Dimensões } \\
\text { externas ao } \\
\text { grupo de } \\
\text { inovação }\end{array}$} & D8 & $\begin{array}{c}\text { Dependência } \\
\text { de recursos } \\
\text { externos }\end{array}$ & $\begin{array}{l}\text { Grau com que um grupo de inovação percebe a sua } \\
\text { dependência de recursos (dinheiro, informação, } \\
\text { materiais etc.) de outros grupos da organização. } \\
\text { AUTORES: Lawrence; Lorsch (1967); Lawrence; Dyer } \\
\text { (1983). }\end{array}$ \\
\hline & D9 & Formalização & $\begin{array}{l}\text { Grau com que os relacionamentos com outros grupos } \\
\text { são verbalizados ou documentados. } \\
\text { AUTORES: Van de Ven (1976). }\end{array}$ \\
\hline & \multirow[t]{2}{*}{ D10 } & \multirow[t]{2}{*}{ Efetividade } & $\begin{array}{l}\text { Grau com que os indivíduos percebem que o relacio- } \\
\text { namento com grupos externos vale a pena e satisfaz } \\
\text { aos objetivos propostos e o grau de responsabilidade } \\
\text { e comprometimento com a inovação. } \\
\text { AUTORES: Blake e Mouton (1964); Burke (1970); } \\
\text { McGrath (1984). }\end{array}$ \\
\hline & & & $\begin{array}{l}\text { Grau com que as partes do relacionamento mudam ou } \\
\text { afetam, ou influenciam umas às outras. } \\
\text { AUTORES: Blake e Mouton (1964); Burke (1970); } \\
\text { McGrath (1984). }\end{array}$ \\
\hline
\end{tabular}

Fonte: Adaptado de Machado e Carvalho (2011)

A partir do Quadro 1, pode-se perceber que o desempenho (resultado) de organizações inovadoras está embasado em uma cultura organizacional voltada para inovação. Esta cultura fomenta a interação, proporcionando uma estrutura que viabilize a utilização de recursos físicos, financeiros e humanos para apoiar e oferecer um clima de criatividade e inovação(AHMED,1998a;AHMED,1998b). Dessa forma, levanta-se a seguinte hipótese: $\mathbf{H}_{3}=\mathrm{O}$ ambiente externo ao grupo de inovação influencia positivamente o ambiente interno ao grupo de inovação.

Em ambos os contextos (tanto interno quanto externo), quando devidamente delineados em um processo de inovação, estes impactam de forma positiva o resultado da organização. Para Van de Ven (1986, p. 591), “[...] o processo de inovação é definido como o desenvolvimento e a implementação de novas ideias por pessoas que, ao longo do tempo, se envolvem em transações com outras, dentro de um contexto institucional." Assim, levanta-se a seguinte hipótese: $\mathbf{H}_{4}=\mathrm{O}$ ambiente interno ao grupo de inovação influencia positivamente o resultado.

Um ambiente favorável ao desenvolvimento de inovação depende ainda de artefatos básicos, como fontes técnicas, sendo composto por pessoas, equipamentos, conhecimentos, recursos financeiros etc., e as competências organizacionais para gerenciá-las. Faz-se necessário ainda um contexto organizacional que favoreça e estimule a criação de novas ideias, para a liberdade de criação e envolvimento entre os funcionários, a fim de que possam gerar e disseminar a inovação (TIDD et al., 1997; AHMED,1998a). Todos esses elementos estão interligados e a liderança organizacional faz 
parte desse contexto, uma vez que ela fornece suporte para a inovação, possibilita o comprometimento dos funcionários e modela a cultura organizacional, por meio de normas e comportamentos que estimulam a aceitação em assumir riscos e tenham uma visão de longo prazo. Os líderes precisam ainda fornecer recursos e manter estruturas adequadas para que a inovação aconteça (ZIGER; MADIQUE, 1990; AHMED, 1998a).

Diante do exposto, pode- se perceber que a capacidade de inovação está relacionada a uma cultura que favoreça $o$ desenvolvimento de inovação, ao conhecimento gerado por meio da participação dos funcionários (relacionamento). Além disso, oferecer ainda um ambiente livre de punições quando uma ideia falha, aprendendo assim com os próprios erros e oferecendo estímulos, para que novas ideias sejam geradas.

Conforme já mencionado, o ambiente inovativo compreende os fatores externos ao grupo de inovação, por meio da formalização e da efetividade de relacionamentos (JAMBULINGAM et al., 2005; AKMAN; YILMAZ, 2008).

\section{ASPECTOS METODOLÓGICOS}

Para responder ao objetivo deste estudo, que foi analisar a influência da cultura organizacional sobre o ambiente de inovação no contexto de uma multinacional varejista, adotou-se uma abordagem quantitativa, com o uso da técnica de pesquisa descritiva com corte transversal. De acordo com Hair Jr. et. al. (2005), a pesquisa descritiva normalmente detalha alguma situação, sendo estruturada e criada especialmente para mensurar as características de eventos ou atividades de pesquisa. Optou-se pelo corte transversal com survey ${ }^{1}$, por proporcionar a coleta dos dados em um determinado espaço de tempo e a sintetização estatística das informações (HAIR Jr. et al., 2005).

A população foi composta pelos funcionários de uma empresa do ramo alimentício pertencente a um grupo multinacional varejista americano

\footnotetext{
${ }^{1}$ O método de pesquisa survey é quantitativo. Sua aplicação é desejada quando o pesquisador pretende investigar o que, porque, como ou quanto se dá determinada situação, não sendo possível através do método, determinar variáveis dependentes e independentes.
}

com filiais no Brasil.A escolha por esta empresa se deu pela acessibilidade aos sujeitos sociais da pesquisa. Optou-se pela amostragem aleatória simplificada, em que se teve acesso a todos os elementos da população e foram selecionados de forma aleatória os respondentes (BARBETTA, 2012). Dessa forma, a pesquisa foi dirigida aos funcionários de todos os departamentos da organização, e foram obtidos um total de 156 questionários válidos.

QuANTO AO INSTRUMENTO

UTILIZADO PARA A COLETA

DE DADOS, OPTOU-SE PELO

QUESTIONÁRIO ADAPTADO

DE BATES ET AL. (1995), NO

QUE SE REFERE À CULTURA

ORGANIZACIONAL.

Este instrumente é dividido em duas dimensões, sendo elas: distância do poder e individualismo versus coletivismo. Utilizou-se 
também o questionário de Machado, Carvalho e Heinzmann (2012) referente ao ambiente de inovação. O instrumento totalizou 39 questões. As questões estão em escala Likert de 7 pontos, com variância entre 1 , que é discordo totalmente e 7, concordo totalmente. O Quadro 2 apresenta as dimensões e os indicadores utilizados neste estudo.

Quadro 2: Dimensões e indicadores da Cultura Organizacional e do Ambiente de Inovação

\begin{tabular}{|c|c|}
\hline \multicolumn{2}{|r|}{ DIMENSÕES E INDICADORES DA CULTURA ORGANIZACIONAL } \\
\hline \multicolumn{2}{|r|}{ DISTÂNCIA DO PODER } \\
\hline Indicadores & Fatores que compõem a dimensão \\
\hline Distância1 & $\begin{array}{l}\text { Pessoas em posições mais elevadas na hierarquia da empresa devem tomar } \\
\text { decisões sem consultar as pessoas em posições inferiores na hierarquia da } \\
\text { empresa. }\end{array}$ \\
\hline Distância2 & $\begin{array}{l}\text { Pessoas em posições mais altas na hierarquia da empresa devem evitar a } \\
\text { interação social com pessoas em posições inferiores na hierarquia da empresa. }\end{array}$ \\
\hline Distância3 & $\begin{array}{l}\text { Pessoas em posições mais baixas na hierarquia da empresa não devem discor- } \\
\text { dar das decisões tomadas por pessoas em posições mais altas na hierarquia } \\
\text { da empresa. }\end{array}$ \\
\hline
\end{tabular}

\section{COLETIVISMO}

\begin{tabular}{|c|l|}
\hline Indicadores & \multicolumn{1}{c}{ Fatores que compõem a dimensão } \\
\hline Coletivismo1 & $\begin{array}{l}\text { As pessoas devem ficar com o grupo mesmo quando o grupo estiver com } \\
\text { dificuldades. }\end{array}$ \\
\hline Coletivismo2 & As chefias encorajam seus subordinados a trabalharem em grupo. \\
\hline Coletivismo3 & $\begin{array}{l}\text { Esta unidade é um bom lugar para as pessoas que gostam de tomar decisões } \\
\text { em grupo. }\end{array}$ \\
\hline Coletivismo4 & $\begin{array}{l}\text { Qualidade da participação do grupo é uma parte significativa na avaliação } \\
\text { do desempenho da chefia. }\end{array}$ \\
\hline
\end{tabular}

DIMENSÕES E INDICADORES DO AMBIENTE DE INOVAÇÃO (MACHADO;

CARVALHO, 2011; 2013)

\section{RESULTADOS}

\begin{tabular}{|c|l|}
\hline Indicadores & Fatores que compõem a dimensão \\
\hline Resultados1 & O resultado final desta ideia me deixou satisfeito. \\
\hline Resultados2 & Odesenvolvimento pessoal que obtive por meio desta ideia me deixou satisfeito. \\
\hline Resultados3 & Os benefícios à instituição gerados por essa ideia me deixaram satisfeito. \\
\hline
\end{tabular}




\section{PROCESSOS}

\begin{tabular}{|c|l|}
\hline Indicadores & \multicolumn{1}{c}{ Fatores que compõem a dimensão } \\
\hline Processos1 & $\begin{array}{l}\text { Os passos corretos e necessários para executar a ideia foram divulgados com } \\
\text { antecedência. }\end{array}$ \\
\hline Processos2 & Eu sabia quais tarefas precisaria realizar para desenvolver essa ideia. \\
\hline Processos3 & $\begin{array}{l}\text { Existiam regras, manuais ou procedimentos que indicavam como trabalho } \\
\text { deveria ser desenvolvido por mim. }\end{array}$ \\
\hline Processos4 & $\begin{array}{l}\text { Durante a fase de desenvolvimento da ideia, foi percebido apoio de pessoas } \\
\text { importantes para sua execução. }\end{array}$ \\
\hline
\end{tabular}

\section{RECURSOS}

\begin{tabular}{|c|l|}
\hline Indicadores & \multicolumn{1}{c|}{ Fatores que compõem a dimensão } \\
\hline Recursos1 & Para realização da ideia, necessitei de recursos financeiros. \\
\hline Recursos2 & Para realização da ideia, necessitei de materiais, espaço e equipamentos. \\
\hline Recursos3 & Para realização da ideia, necessitei de atenção das chefias. \\
\hline Recursos4 & Para realização da ideia, necessitei de pessoas. \\
\hline
\end{tabular}

\section{LIDERANÇA}

\begin{tabular}{|c|l|}
\hline Indicadores & \multicolumn{1}{c}{ Fatores que compõem a dimensão } \\
\hline Liderança1 & $\begin{array}{l}\text { As pessoas que trabalham na Instituição não são repreendidas ou criticadas } \\
\text { quando não atingem os objetivos definidos no desenvolvimento de uma nova } \\
\text { ideia. }\end{array}$ \\
\hline Liderança2 & $\begin{array}{l}\text { As pessoas que lideram a execução de uma nova ideia se preocupam com o } \\
\text { bom relacionamento do grupo. }\end{array}$ \\
\hline Liderança3 & $\begin{array}{l}\text { Ainstituição valoriza as pessoas que tentam fazer algo diferente para melhorar } \\
\text { o processo mesmo quando há eventuais erros. }\end{array}$
\end{tabular}

\section{AUTONOMIA}

\begin{tabular}{|c|l|}
\hline Indicadores & \multicolumn{1}{|c|}{ Fatores que compõem a dimensão } \\
\hline Autonomia1 & $\begin{array}{l}\text { Eu pude opinar, participar ou dar palpites sobre a necessidade de chamar } \\
\text { pessoas de outras áreas para ajudar no desenvolvimento desta inovação. }\end{array}$ \\
\hline Autonomia2 & $\begin{array}{l}\text { Eu participei, opinei ou ajudei a definir os recursos financeiros que seriam } \\
\text { utilizados para o desenvolvimento da ideia. }\end{array}$ \\
\hline Autonomia3 & $\begin{array}{l}\text { No dia a dia do trabalho, sinto-me à vontade para falar o que penso sobre } \\
\text { minhas ideias. }\end{array}$ \\
\hline
\end{tabular}




\begin{tabular}{|c|c|}
\hline \multicolumn{2}{|r|}{ RELACIONAMENTO INTERNO DO GRUPO DE INOVAÇÃO } \\
\hline Indicadores & Fatores que compõem a dimensão \\
\hline Relac.interno1 & $\begin{array}{l}\text { Frequentemente eu era comunicado por outras pessoas sobre problemas } \\
\text { ocorridos no processo da inovação. }\end{array}$ \\
\hline Relac.interno2 & $\begin{array}{l}\text { Quando tenho alguma ideia, sou incentivado a trabalhar em parceria com } \\
\text { outras pessoas. }\end{array}$ \\
\hline Relac.interno3 & $\begin{array}{l}\text { Os desentendimentos ocorridos no desenvolvimento de uma ideia são } \\
\text { considerados importantes e logo são resolvidos. }\end{array}$ \\
\hline \multicolumn{2}{|r|}{ RELACIONAMENTO EXTERNO AO GRUPO DE INOVAÇÃO } \\
\hline Indicadores & Fatores que compõem a dimensão \\
\hline Relac.externo1 & $\begin{array}{l}\text { Os trabalhos realizados em parceria com outros grupos poderão ser utilizados } \\
\text { no desenvolvimento de novas ideias. }\end{array}$ \\
\hline Relac.externo2 & $\begin{array}{l}\text { Não houve discussões nem desentendimento entre as pessoas envolvidas na } \\
\text { execução da ideia. }\end{array}$ \\
\hline Relac.externo3 & $\begin{array}{l}\text { Houve o apoio de pessoas qualificadas de outras áreas da instituição, que } \\
\text { ajudaram no processo de desenvolvimento ou implantação da inovação. }\end{array}$ \\
\hline \multicolumn{2}{|r|}{ DEPENDÊNCIA DE RECURSOS EXTERNOS } \\
\hline Indicadores & Fatores que compõem a dimensão \\
\hline Dep.Recursos 1 & $\begin{array}{l}\text { Eu e meu grupo precisamos de apoio, ajuda e informações de outras pessoas } \\
\text { ou grupos que trabalham na instituição para desenvolver a ideia. }\end{array}$ \\
\hline Dep.Recursos2 & $\begin{array}{l}\text { Uma parte do trabalho que deveria ser feito por mim ou pelo meu grupo } \\
\text { para o desenvolvimento da ideia foi realizado por outra pessoa ou grupo da } \\
\text { instituição. }\end{array}$ \\
\hline Dep.Recursos3 & $\begin{array}{l}\text { Eu e meu grupo precisamos do auxílio de outras pessoas e grupos da instituição } \\
\text { para a obtenção de recursos materiais para o desenvolvimento e implantação } \\
\text { da inovação. }\end{array}$ \\
\hline \multicolumn{2}{|r|}{ FORMALIZAÇÃO } \\
\hline Indicadores & Fatores que compõem a dimensão \\
\hline Formalização1 & $\begin{array}{l}\text { Conversas e discussões entre o meu e outros grupos que trabalham na insti- } \\
\text { tuiçãa auxiliaram no nosso relacionamento e no desenvolvimento de ideias. }\end{array}$ \\
\hline Formalização2 & $\begin{array}{l}\text { Documentos e anotações foram feitos explicando como a parceria entre o meu } \\
\text { e outros grupos que trabalham na instituição auxiliariam no desenvolvimento } \\
\text { da ideia. }\end{array}$ \\
\hline Formalização3 & $\begin{array}{l}\text { Durante o desenvolvimento da inovação, houve uma sequência de comunicação } \\
\text { entre mim e meu grupo com pessoas de outras áreas da instituição que também } \\
\text { se envolveram com esta inovação. }\end{array}$ \\
\hline
\end{tabular}




\begin{tabular}{|c|c|}
\hline \multicolumn{2}{|c|}{ EFETIVIDADE DO RELACIONAMENTO } \\
\hline Indicadores & Fatores que compõem a dimensão \\
\hline $\begin{array}{c}\text { Efet. } \\
\text { relacionamento1 }\end{array}$ & Sinto-me satisfeito com as parcerias firmadas no desenvolvimento da ideia. \\
\hline $\begin{array}{c}\text { Efet. } \\
\text { relacionamento2 }\end{array}$ & $\begin{array}{l}\text { Os compromissos assumidos por outros grupos que trabalham na instituição } \\
\text { foram cumpridos no desenvolvimento da ideia. }\end{array}$ \\
\hline $\begin{array}{c}\text { Efet. } \\
\text { relacionamento3 }\end{array}$ & $\begin{array}{l}\text { Eu e meu grupo conseguimos alterar facilmente, quando necessário, o trabalho } \\
\text { de outros grupos envolvidos no desenvolvimento da ideia. }\end{array}$ \\
\hline
\end{tabular}

Fonte: Adaptado de Hofstede (1980); Bates et al. (1995); Machado, Carvalho e Heinzmann (2012).

Quanto à coleta dos dados, o questionário foi impresso e aplicado pelos autores pessoalmente nas duas unidades da empresa e ocorreu no período de março a abril de 2014. Quanto à técnica para análise dos dados, utilizou-se a modelagem de equações estruturais. De acordo com Marôco (2010), a análise de equações estruturais é uma técnica utilizada para testar a validade de modelos teóricos que definem relações causais e hipotéticas entre variáveis. Esta técnica foi adotada a fim de analisar a influência da cultura sobre o ambiente de inovação das empresas estudadas.

Os dados levantados foram tabulados em planilha do software Microsoft Excel $^{\circledR}$. As questões foram agrupadas às referidas dimensões, descritas no Quadro 2. Cada dimensão alcançou uma média correspondente à soma das respostas das questões, divididas por dimensão e por respondente, e dividida pela quantidade de questões de cada dimensão do questionário.

A base de dados da planilha do software Microsoft Excel ${ }^{\oplus}$ foi importada para o Software Estatístico SPSS ${ }^{\circledast}$ (Statistical Package for the Social Sciences), versão 22, e AMOS ${ }^{\circledast}$, versão 22. A primeira análise realizada foi o teste de confiabilidade (Alfa de Cronbach). Este indicador é uma medida de confiabilidade que varia de 0 a 1 , sendo os valores de 0,60 a 0,70 considerados o limite inferior de aceitabilidade (HAIR Jr. et al., 2009). Depois foi realizada a técnica estatística de modelagem de equações estruturais, em que foi proposto um modelo (Figura 1) e a análise dos índices das questões/ dimensões: $t$-value, $p$-value e $R 2$.

Conforme Koufteros (1999), o t-value são considerados aceitáveis quando seus valores são maiores do que $<2$ ou $<2,576$ e considerados significativos ao nível de 0,05 e 0,01, respectivamente. $\mathrm{O}$ índice $p$-value é significativo se for diferente de zero ao nível de 0,01. O R ${ }^{2}$ é adequado acima de 0,5. Quanto aos índices do modelo, segundo Hair Jr. et al. (2009), para obterem significância estatística, o $x^{2}$ (CMIN) deve ter o valor abaixo de 0,05. Quanto ao GFI (Índice de Qualidade de Ajuste), o intervalo possível de valores é de 0 a 1 , em que valores maiores que 0,9 são considerados bons. O RMSEA (raiz do erro quadrático médio de aproximação), para ser aceitável, deve estar abaixo de $0,10 . \mathrm{OCFI}$ (índice de ajuste comparativo) é um índice de ajuste incremental. Seus valores variam entre 0 e 1 , em que valores mais altos indicam um melhor ajuste. Já valores abaixo de 0,90 não são, geralmente, associados a um modelo que se ajusta bem. Após a descrição da metodologia utilizada na pesquisa em questão, apresentam-se, no capítulo 4, os resultados obtidos e as análises dos mesmos . 


\section{APRESENTAÇÃO E ANÁLISE DOS RESULTADOS}

Dando sequência ao estudo, nesta etapa serão apresentados e analisados os dados obtidos para a pesquisa.Com relação ao grau de instrução dos respondentes, a Tabela 1 representa a formação acadêmica dos funcionários entrevistados na pesquisa.

Tabela 1: Formação acadêmica

\begin{tabular}{c|c|c}
\hline Titulação & Frequência & $\%$ \\
\hline Ensino Fundamental & 7 & 4,5 \\
\hline Ensino Médio & 55 & 35,3 \\
\hline Superior Completo & 69 & 44,2 \\
\hline Superior Incompleto & 17 & 10,9 \\
\hline Especialização & 8 & 5,1 \\
\hline Total & $\mathbf{1 5 6}$ & $\mathbf{1 0 0 , 0}$ \\
\hline
\end{tabular}

Fonte: Dos autores (2014)

No que diz respeito à formação educacional, pode-se perceber, de acordo com a Tabela 1, que dos 156 respondentes, 69 possuem Ensino Superior completo, representando a maioria dos entrevistados. Na sequência, estão os que possuem o Ensino Médio completo, sendo representado por 55 funcionários. Dos respondentes, destacam-se ainda 17 que não completaram o Ensino Superior ou estão cursando, além dos 07 que tem até o Ensino Fundamental completo.
No que se refere ao gênero dos
entrevistados, a maioria é do gênero
feminino, representando 86 dos 156
empregados, contra 70 do gênero
masculino. A faixa etária dos colaboradores
varia de 17 a 61 anos.

Em relação à idade dos respondentes, a maioria é da faixa etária de 20 a 40 anos, totalizando 123 respondentes, o que corresponde a 78,7\% dos entrevistados. Vale ressaltar ainda que a média de idade dos respondentesé de 33 anos. Quanto ao estado civil, a pesquisa revelou que a maioria é solteira, sendo representada por $39,1 \%$, os empregados que possuem uma relação conjugal estável são de $32,17 \%$ e os que são viúvos ou separados somam juntos $28,73 \%$.

Em relação ao modelo estrutural proposto para este estudo (Figura 1), a questão coletivismo (M_CO) influencia a dimensão do ambiente interno e do ambiente externo, que, por sua vez, influência a dimensão do ambiente interno. Por último, a dimensão do ambiente interno influencia a dimensão dos resultados. 
Depois da especificação do modelo, é necessário verificar a sua validade de medida, ou seja, se o modelo proposto para mensuração é válido, esta validade dependerá da qualidade do ajuste realizado (HAIR Jr. et al., 2009). Nesse ínterim, na Tabela 2, é possível verificar que os índices das cargas das questões contidas nas dimensões, antes da depuração, possuíam valores que não correspondiam aos ajustes que estabeleciam a aceitabilidade de alguns índices ( $t$-value, $p$-value, $\mathrm{R}^{2}$ ) para o modelo, segundo Koufteros (1999) e Hair Jr. et al. (2009).

Tabela 2: Cargas não padronizadas e padronizadas

\begin{tabular}{|c|c|c|c|c|c|c|c|}
\hline Dimensões & Questões & $\begin{array}{c}\text { Carga não } \\
\text { padronizada }\end{array}$ & $\begin{array}{c}\text { Carga } \\
\text { padronizada }\end{array}$ & $\begin{array}{l}\text { S.E. } \\
\text { (erro) }\end{array}$ & $\begin{array}{c}\text { C.R } \\
\text { (t-value })\end{array}$ & $\begin{array}{c}\mathbf{P} \\
(p-\text { value })\end{array}$ & $\mathbf{R}^{2}$ \\
\hline \multirow{3}{*}{$\begin{array}{c}\text { Ambiente } \\
\text { Externo }\end{array}$} & $\frac{M_{-}}{\text {INOVFORM }}$ & 1 & 0,731 & & & & 0,53 \\
\hline & $\frac{M_{-}}{\text {INOVDEPEX }}$ & 0,751 & 0,592 & 0,11 & 6,978 & $* * *$ & 0,35 \\
\hline & M_INOVEFE & 1,007 & 0,784 & 0,11 & 9,245 & $* * *$ & 0,61 \\
\hline \multirow{6}{*}{$\begin{array}{c}\text { Ambiente } \\
\text { Interno }\end{array}$} & M_INOVP & 1 & 0,772 & & & & 0,60 \\
\hline & M_INOVREC & 0,572 & 0,44 & 0,11 & 5,352 & $* * *$ & 0,19 \\
\hline & M_INOVLI & 1,022 & 0,677 & 0,12 & 8,567 & $* * *$ & 0,46 \\
\hline & M_INOVAUT & 0,994 & 0,741 & 0,11 & 9,499 & $* * *$ & 0,55 \\
\hline & M_INOVREL & 0,97 & 0,734 & 0,1 & 9,398 & $* * *$ & 0,54 \\
\hline & $\frac{M_{-}}{\text {INOVRELEX }}$ & 0,625 & 0,507 & 0,1 & 6,229 & $* * *$ & 0,26 \\
\hline \multirow{2}{*}{ Cultura } & $\begin{array}{c}\text { M_CO } \\
\text { (coletivismo) }\end{array}$ & 1 & 0,558 & & & & 0,31 \\
\hline & M_DP & $-0,396$ & $-0,209$ & 0,18 & $-2,265$ & 0,02 & 0,04 \\
\hline \multirow{3}{*}{ Resultados } & INOVR8 & 1 & 0,692 & & & & 0,48 \\
\hline & INOVR9 & 1,122 & 0,83 & 0,14 & 8,018 & $* * *$ & 0,69 \\
\hline & INOVR10 & 0,958 & 0,714 & 0,13 & 7,486 & $* * *$ & 0,51 \\
\hline
\end{tabular}

*** Indica que a questão é significativamente diferente de zero ao nível de 0,01.

Índices de Ajuste: $\mathrm{X} 2=181,431, \mathrm{df}=72, \mathrm{X} 2 / \mathrm{df}=2,520, \mathrm{GFI}=0,855, \mathrm{CFI}=0,872, \mathrm{RMSEA}=0,099$

Fonte: Dos autores (2014) 
Dentre as questões/dimensões que necessitam destes ajustes está a Dependência de recursos externos (M_INOVDEPEX) na dimensão do Ambiente Externo, em que o $t$-value é de 6,978, o p-value é significante (diferente de zero ao nível de 0,01$)$ e o $\mathrm{R}^{2}$ é de 0,35 . Em seguida, a outra questão foi Recursos (M_INOVREC) na dimensão do Ambiente Interno, em que o t-valueé de 5,352, o p-valueé significante (diferente de zero ao nível de 0,01) e o $\mathrm{R}^{2}$ é de 0,19. Aquestão seguinte foi Relacionamento externo ao grupo de inovação (M_INOVRELEX), em que o $t$-valueé de 6,229, o p-value é significante (diferente de zero ao nível de 0,01 ) e o $\mathrm{R}^{2}$ é de 0,26. Por último, na dimensão de cultura, está a questão Distância do Poder (M_DP), em que o t-value é de -2,265, o p-value não foi significante $(0,02)$ e o $\mathrm{R}^{2}$ de 0,04 .

Quanto aosíndices do modelo (X2 =181,431,df $=72, \mathrm{X} 2 / \mathrm{df}=2,520, \mathrm{GFI}=0,855, \mathrm{CFI}=0,872$, RMSEA =0,099), estes não estavam aceitáveis para o modelo proposto.Assim, conforme indica a teoria, a depuração das questões/dimensões foi realizada para melhorar os índices do modelo, conforme apresentado na Tabela 3 (HAIR Jr. et al., 2009).

Tabela 3: Cargas não padronizadas e padronizadas (depuração da dimensão de cultura)

\begin{tabular}{|c|c|c|c|c|c|c|c|}
\hline Dimensões & Questões & $\begin{array}{c}\text { Carga não } \\
\text { padronizada }\end{array}$ & $\begin{array}{c}\text { Carga } \\
\text { padronizada }\end{array}$ & $\begin{array}{c}\text { S.E. } \\
\text { (erro) }\end{array}$ & $\begin{array}{c}\text { C.R } \\
\text { (t-value })\end{array}$ & $\begin{array}{c}\mathbf{P} \\
(p \text {-value })\end{array}$ & $\mathbf{R}^{2}$ \\
\hline \multirow{2}{*}{$\begin{array}{l}\text { Ambiente } \\
\text { Externo }\end{array}$} & $\frac{M_{-}}{\text {INOVFORM }}$ & 1 & 0,765 & & & & 0,59 \\
\hline & M_INOVEFE & 0,952 & 0,776 & 0,102 & 9,288 & $* * *$ & 0,6 \\
\hline \multirow{4}{*}{$\begin{array}{l}\text { Ambiente } \\
\text { Interno }\end{array}$} & M_INOVP & 1 & 0,785 & & & & 0,62 \\
\hline & M_INOVLI & 1,044 & 0,704 & 0,117 & 8,938 & $* * *$ & 0,5 \\
\hline & M_INOVAUT & 0,903 & 0,685 & 0,105 & 8,632 & $* * *$ & 0,47 \\
\hline & M_INOVREL & 0,863 & 0,665 & 0,103 & 8,343 & $* * *$ & 0,44 \\
\hline \multirow{3}{*}{ Resultados } & INOVR8 & 1 & 0,692 & & & & 0,48 \\
\hline & INOVR9 & 1,111 & 0,822 & 0,140 & 7,954 & $* * *$ & 0,68 \\
\hline & INOVR10 & 0,967 & 0,721 & 0,129 & 7,502 & $* * *$ & 0,52 \\
\hline Cultura & M_CO & 1 & 1 & - & - & - & - \\
\hline *** Indica que & stão é signif & ente di & $2 \pi$ & de $0, c$ & 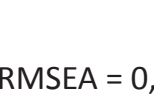 & 98 & \\
\hline
\end{tabular}

Fonte: Dos autores (2014) 
Conforme a Tabela 3, alguns indicadores apresentaram $\mathrm{R}^{2}$ menor do que o sugerido pela literatura, porém optou-se por mantê-los, a fim de satisfazer o número mínimo de indicadores recomendado para cada dimensão. Exceto a dimensão cultura, que era composta por dois indicadores, que, no caso de não aderência de um desses, converteu-se o indicador mais significante em variável que representa a dimensão. Desta forma, os modelos de medidas, após a purificação dos dados, chegaram aos índices de ajuste sugeridos pela literatura pertinente. Isso posto, deu-se continuidade às análises por meio do teste do modelo estrutural, conforme as hipóteses descritas no decorrer deste estudo.

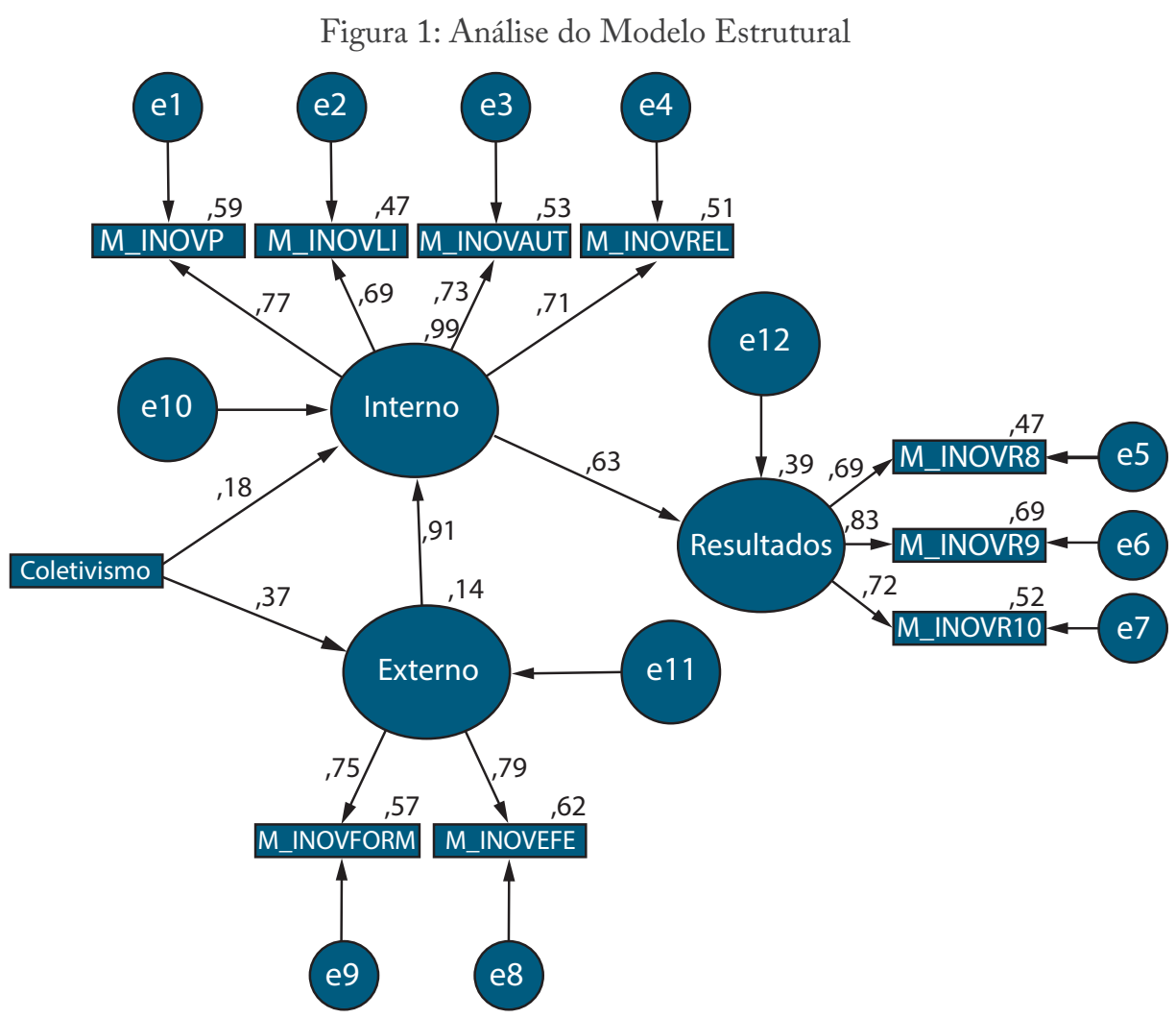

Fonte: Dos autores (2014)

Com base nas cargas fatoriais

padronizadas, averiguou-se a validade das relações propostas nos constructos de segunda ordem: interno, externo e resultados.

De acordo com a Figura 1, a variável coletivismo influencia $\lambda=0,37$ na variável do ambiente externo e $\lambda=0,18$ no ambiente interno. Além do mais, salienta-se que houve validade das relações propostas no constructo de segunda ordem (interno) com as variáveis de primeira ordem: processos: M_INOVP $(\lambda=0,77)$; liderança: M_INOVLI $(\lambda=0,69)$; autonomia:M_INOVAUT $(\lambda=0,73)$; relacionamento interno do grupo de inovação: M_INOVREL $(\lambda=0,71)$; validade das relações propostas no constructo de segunda ordem (externo) com as variáveis de primeira ordem: formalização: $\mathrm{M}_{-}$ INOVFORM $(\lambda=0,75)$; efetividade do relacionamento:M_INOVEFE $(\lambda=0,79)$.Verifica-se, portanto, que o coletivismo influencia mais o ambiente externo do que o interno. Levando em consideração ainda a variável coletivismo e o ambiente externo, estas geram no ambiente interno um coeficiente de determinação $\left(\mathrm{R}^{2}\right) \mathrm{de}$ 
0,99, e a variável coletivismo causa no ambiente externo um coeficiente de determinação $\left(\mathrm{R}^{2}\right)$ de 0,14 . Este dado demonstra a influência que o coletivismo e o ambiente externo possuem sobre o ambiente interno. Sob essa perspectiva, confirmam-se as hipóteses: $\mathbf{H}_{\mathbf{1}}=$ a cultura influencia positivamente o ambiente interno ao grupo de inovação e $\mathbf{H}_{2}=$ a cultura influencia positivamente o ambiente externo ao grupo de inovação.

De acordo com Hofstede (1980; 1990) e Pettigrew (1982), a cultura coletivista é baseada em uma gestão de grupos, valorizando as oportunidades de treinamento externo ao seu grupo, contemplando as habilidades dos indivíduos. Para Hofstede (1980; 1990), o interesse do grupo prevalece sobre o interesse do indivíduo. Tanto para Hofstede (1980; 1990) quanto para Pettigrew (1982), os estudos acerca da cultura das organizações oferecem axiomas de que os valores culturais são influenciados pelo compartilhamento de informações e conhecimentos entre seus indivíduos. A cultura, por sua vez, promove um contexto organizacional que possibilita essa interação social. Assim, a cultura influi na forma como os colaboradores se comunicam e compartilham seus conhecimentos na empresa.

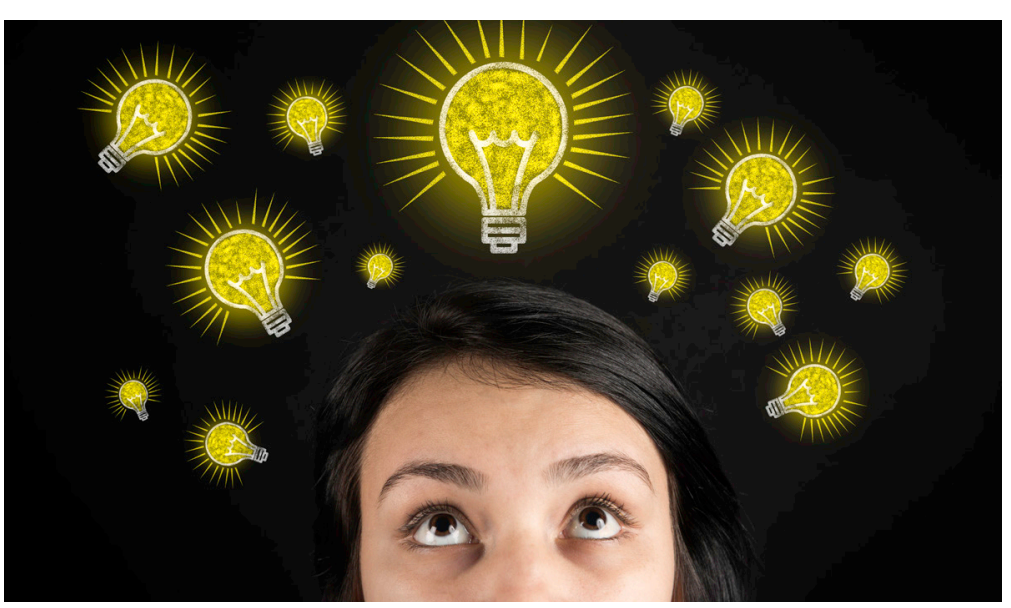

Nesta perspectiva, quando a organização tem uma cultura congruente, incentiva o coletivismo e atua com uma pequena distância de poder. Percebe-se que a parceria com outros grupos é válida e importante para o desenvolvimento de uma nova ideia, propiciando um favorecimento ao ambiente de inovação (VAN DE VEN; CHU, 1989; MACHADO; CARVALHO, 2011). Jambulingam et al. (2005) e Akman e Yilmaz (2008) declaram que a capacidade de uma organização em inovar está fundamentalmente relacionada à cultura de inovação dessa empresa, ao conhecimento gerado pelos indivíduos que nela atuam, às ideias que emergem, assim como aos seus processos internos e à adequação às mudanças que resultam no ambiente.Van de Ven (1989) complementa que a inovação não é um processo a ser realizado de forma individual, ou setorialmente, mas sim por um grupo de pessoas que cooperam e que estejam comprometidas, com o intuito de desenvolver ideias por meio destas interações, auferindo a aceitação dessas ideias e buscando a legitimidade.

Quanto a influência da variável do ambiente externo ao grupo de inovação sobre o ambiente interno, (Figura 1), constata-se que o ambiente externo influencia consideravelmente $(\lambda=0,91)$ a variável do ambiente interno. Assim, confirma-se a seguinte hipótese: $\mathbf{H}_{3}=$ o ambiente externo ao grupo de inovação influencia positivamente o ambiente interno ao grupo de inovação. Segundo Van de Ven e Chu (1989), esta dimensão é caracterizada pelo grau de percepção dos colaboradores do grupo de inovação sobre a necessidade de envolvimento com indivíduos externos ao grupo de inovação, a fim de obter materiais, recursos, informações, entre outros. Estes fatores externos resultam na influência ao ambiente interno. Esta interação pode converter-se em novas ideias e suscitar a inovação. 
Segundo Jambulingam et al. (2005) e Akman e Yilmaz (2008), a administração da inovação ocorre tanto nos ambientes internos quanto externos. A inovação no âmbito interno está relacionada aos processos de identificação e construção de competências elementares, à codificação e à transferência de conhecimento, à identificação de oportunidades e à aplicação de uma estratégia capaz de integrar os processos organizacionais. Já no âmbito externo, a inovação está relacionada à aquisição de recursos financeiros e à interação com outros grupos, como áreas de marketing, administrativas, manutenção, entre outras, a fim de contribuírem para a disseminação e criação de conhecimento.

Com relação à variável resultados, verifica-se (Figura 1) que houve validade das relações propostas no constructo de segunda ordem (resultados) com as variáveis de primeira ordem: satisfação com o resultado final da ideia: INOVR8 $(\lambda=0,69)$; satisfação com o desenvolvimento pessoal obtido por meio da ideia: INOVR9 $(\lambda=0,83)$; a satisfação com os benefícios que a empresa obteve gerados pela ideia: INOVR10 $(\lambda=0,72)$. Ainda, quanto ao constructo de segunda ordem resultados, constata-se que o mesmo é influenciado pelo constructo do ambiente interno $(\lambda=0,63)$, gerando um coeficiente de determinação $\left(\mathrm{R}^{2}\right)$ de 0,39. Dessa forma, confirma-se a última hipótese proposta neste artigo: $\mathbf{H}_{4}=\mathrm{o}$ ambiente interno ao grupo de inovação influencia positivamente o resultado.

Neste estudo, a variável resultados é representada pela satisfação dos funcionários da organização em relação ao resultado que estes obtiveram naimplantação da ideia gerada.Estes resultados demonstram que os colaboradores perceberam que a inovação atendeu às suas perspectivas individuais e da organização, no que se refere aos resultados. Neste sentido, Machado e Carvalho (2011) ressaltam que, quanto maior a percepção da efetividade da inovação por parte da organização e de seus empregados, maior será a percepção e a sua caracterização como sendo um ambiente inovador. Assim, é possível inferir, com base nesta dimensão, que o ambiente de inovação é percebido pela organização. Dessa forma, o ambiente interno influencia positivamente o resultado preterido pelas empresas.

\section{CONCLUSÃO}

A pesquisa teve como principal objetivo analisar a influência da cultura organizacional sobre o ambiente de inovação no contexto de uma empresa multinacional varejista no Brasil.
Os resultados empíricos obtidos neste estudo demonstraram que houve relação positiva quanto à influência da cultura sobre o ambiente de inovação, tanto em âmbito interno quanto externo ao grupo de inovação.

Assim, a cultura é um fator que determina positivamente as dimensões interna, externa e resultados.

Dessa forma, constatou-se que a cultura influencia consideravelmente o ambiente de inovação, uma vez que houve validade nas relações propostas nos seus constructos com as variáveis: interno - processos, liderança, autonomia, relacionamento interno do grupo de inovação; externo - formalização, efetividade 
do relacionamento.Estes resultados confirmam que essas variáveis afetam positivamente o ambiente de inovação da organização. Quanto à dimensão do ambiente externo e sua influência sobre o ambiente interno, respectivamente, percebe-se que o primeiro possui influência considerável sobre o segundo.

De acordo com Ettlie et al. (1994), o estudo sobre o processo de inovação nos ambientes organizacionais (o ambiente interno e o ambiente externo) desenvolveu-se como um esforço multidisciplinar no decorrer do tempo, considerando a capacidade da organização no desenvolvimento de novos produtos e serviços. A inovação enseja às organizações aprimorarem a qualidade de sua produção, prospectar novos mercados, responder ao avanço da concorrência e alavancar investimentos em novas tecnologias.

Com relação à dimensão resultado, averiguou-se que o ambiente interno influencia positivamente esse constructo (resultado). Quanto ao aprimoramento de um ambiente que seja favorável à inovação, tanto no contexto interno quanto externo, quando estes estão devidamente delineados em um processo de inovação, acabam impactando de forma positiva o resultado da organização.

\section{PODE-SE INFERIR QUE O}

RESULTADO DE EMPRESAS

INOVADORAS SE ALICERÇA

EM UMA CULTURA

ORGANIZACIONAL INCLINADA

PARA INOVAÇÃO, A QUAL

DISPONIBILIZA RECURSOS

QUE APOIAM E OFERECEM UM

AMBIENTE DE CRIATIVIDADE E

INOVAÇÃO.
Considerando os aspectos que constituem o ambiente interno, verifica-se que estes são tão relevantes quando os aspectos que constituem o ambiente externo. Esta relação é tão importante para a manutenção da competitividade organizacional quanto as mudanças provenientes do ambiente (AHMED, 1998a; AHMED, 1998b).

Como limitação desta pesquisa, pode-se destacar a aplicação de uma survey para uma única empresa, impossibilitando a generalização dos resultados por apresentar um diagnóstico enviesado. Outra limitação foi com relação ao questionário, uma vez que os autores do instrumento utilizado na pesquisa de Bates et al. (1995) indicam três dimensões de análise Coletivismo,Distância do poder e Congruência cultural. No entanto, após a análise da técnica fatorial exploratória e confirmatória, foram removidas as dimensões Congruência Cultural e Distância do Poder, por não apresentarem índices estatísticos aceitáveis, permanecendo apenas a dimensão Coletividade. Vale destacar que o estudo possui validade estatística, uma vez que, para a amostra estudada, o modelo não se confirmou com a análise das três dimensões, representando ainda, uma limitação para a pesquisa.

Como implicação acadêmica e gerencial, destaca-se a importância para as organizações em identificarem seus estilos de cultura e como influenciam no ambiente de inovação, para que os objetivos estejam em conformidade com as crenças e valores das organizações. Este estudo contribui para os acadêmicos e estudiosos que buscam disseminar a temática inovação e cultura nas organizações, tendo em vista que, para a empresa ser inovadora, ela necessita que na sua cultura seja disseminado o ambiente inovador. 
Para estudos futuros, sugere-se ampliar a amostra a outras empresas de outros setores da economia, para verificar se o ambiente organizacional impacta na inovação dessas empresas, assim como testar os resultados aqui apresentados no sentido de verificar possíveis extrapolações em um outro contexto. 


\section{CULTURE AND \\ INNOVATION: \\ STUDY ON A \\ MULTINATIONAL \\ RETAILER}

\begin{abstract}
The objective was to analyze the influence of organizational culture on innovation environment in a multinational retailer company. To this end we employed the questionnaire adapted Hofstede (1980) and Bates et al. (1995) for the culture, and the ax; Carvalho; Heinzmann (2012) on the innovation environment. The sample consisted of 156 respondents. Data were analyzed using structural equation modeling. The results indicated that there was a positive relationship in the influence of culture on innovation environment. Forfuture research, it is indicated enlarge the sample to other companies and establish a relationship between innorvation and culture.
\end{abstract}

Keywords: Organizational culture. Innovation environment. Organization. Results.

\section{REFERÊNCIAS}

AFUAH, Allan et al. La dinámica de la innovación organizacional: el nuevo concepto para lograr ventajas competitivas y rentabilidad. Oxford University Press, 1999.

AHMED, P. K. Culture and climate for innovation. European Journal of Innovation Management, v. 1, n. 1, p. 30-43, 1998a.

. Benchmarking innovation best practice.

European Centre of Total Quality Management, v. 5 , n. 1 , p. $45-58,1998$ b.

AKMAN, G., YILMAZ, C. Innovative capability, innovation strategy and Market orientation: na empirical analysis in Turkish software industry. International Journal of Innovation Management, v. 12, n. 1, p. 69-111, 2008.

BALTHAZARD, Pierre A.; COOKE, Robert A. Organizational culture and knowledge management success: assessing the behaviorperformance continuum. In: System Sciences, 2004. Proceedings of the 37 th Annual Hawaii International Conference on. IEEE, p. 10, 2004.

BARBETTA, Pedro Alberto. Estatística aplicada às ciências sociais. 8. ed. rev. Florianópolis: UFSC, 2012.

BARZOTTO, Luciano da Costa. O ambiente de inovação em instituição hospitalar. 2008. 154f. Dissertação (Mestrado em Administração) - Programa de Pós-Graduação em Administração, Universidade Regional de Blumenau, Blumenau, 2008.

BATES, Kimberly A. et al. The crucial interrelationship between manufacturing strategy and organizational culture. Management Science, v. 41, n. 10, p. 1565-1580, 1995.

BERGER, P., LUCKMANN, L. The social construction of reality. New York: Donbleday, 1967.

BOURDIEU, Pierre. O poder simbólico. Rio de Janeiro: Bertrand Brasil, 1989.

CARVALHO, Luciano Castro. Ambiente de inovação: análise multidimensional em uma organização do setor metalomecânico. 2010. Dissertação (Mestrado em Administração) Programa de Pós-Graduação em Administração, Universidade Regional de Blumenau, Blumenau, 2010. 
DAMANPOUR, Fariborz. Organizational complexity and innovation: developing and testing multiple contingency models. Management science, v. 42, n. 5, p. 693-716, 1996.

ETTLIE, John E. et al. Organization strategy and structural differences for radical versus incremental innovation. Management Science, v. 30, n. 6, p. 682695, 1984.

GEORGOPOULOS, Basil Spyros; MANN, Floyd Christopher. The community general hospital. New York: Macmillan, 1962.

GIDDENS, Anthony. The constitution of society: outline of the theory of structuration. Cambridge: Polity Press, 1984.

HAIR JR, J. F. et al. Fundamentos de métodos de pesquisa em administração. Porto Alegre: Bookman, 2005.

Análise multivariada de dados. 6. ed. Porto Alegre: Bookman, 2009.

HANDY, Charles, B. Como compreender as organizações. Rio de Janeiro: Zahar Editores, 1978.

HOFSTEDE, G. Culture's consequences: international differences in work-related values. Beverly Hills, CA: Sage, 1980.

HOFSTEDE, Geert et al. Measuring organizational cultures: a qualitative and quantitative study across twenty cases. Administrative Science Quarterly, v. 35, n. 2, p. 286-316, 1990.

ISIDRO FILHO, Antonio. GUIMARÃES, Tomás de Aquino. Conhecimento, aprendizagem e inovação em organizações: uma proposta de articulação conceitual. Revista de Administração e Inovação, São Paulo, v. 7, n. 2, p. 127-149, abr./jun. 2010.

JAMBULINGAM, T. et al. Entrepreneurial Orientation as a Basis for Classification within a Service Industry: The case of retail pharmacy industry. Journal of Operations Management, v. 23, p. 23-24, 2005.

KANUNGO, Shivraj; SADAVARTI, Sanjeev; SRINIVAS, Yadlapati. Relating IT strategy and organizational culture: an empirical study of public sector units in India. The Journal of Strategic Information Systems, v. 10, n. 1, p. 29-57, 2001.

KOUFTEROS, Xenophon A. Testing a model of pull production: a paradigm for manufacturing research using structural equation modeling. Journal of Operations Management, v. 17, n. 4, p. 467-488, 1999.
MACHADO, D. D. P. N. Inovação e cultura organizacional: um estudo sobre os elementos culturais que interferem no processo de inovação, 2004. Tese de Doutorado (Doutorado em Administração de Empresas) Fundação Getúlio Vargas - SP, FGV-SP, 2004.

MACHADO, D. D. P. N.; HEINZMANN, Lígia Maria; LOESCH, Cláudio. Estratégia de produção e cultura organizacional: podem estar interrelacionadas. Seminário Latino-Iberoamericano de Gestión Tecnológica, v. 13, 2009.

MACHADO, D. P. P. N; CARVALHO, L.

Ambiente favorável ao desenvolvimento de inovações: proposição de um modelo de análise organizacional. In: XXXV Encontro da ANPAD, 2011, Rio de Janeiro. Anais... Rio de Janeiro: Editora da ANPAD, 2011.

MACHADO, Denise Del Prá Netto; CARVALHO, Luciano Castro; HEINZMANN, Lígia Maria.

Ambiente favorável ao desenvolvimento de inovações e cultura organizacional: integração de duas perspectivas de análise. Revista de Administração, v. 47, n. 4, p. 715-729, 2012.

MARÔCO, João. Análise de equações estruturais: fundamentos teóricos, software \& aplicações. Report Number, Lda, 2010.

PÉREZ-BUSTAMANTE, Guillermo. Knowledge management in agile innovative organisations.

Journal of knowledge management, v. 3, n. 1, p. 6-17, 1999.

PETTIGREW, Andrew M. On studying organizational cultures. In: Administrative Science Quarterly, v. 24, n. 4, p. 570-581, 1979.

QUINN, R. E., McGRATH, M. R. H. (1985).

The Transformation of Organizational Cultures: A Competing Values Perspective. In: Organizational Culture, Newvury Park, CA: Sage, 1985.

SCARPIN, Márcia Regina Santiago; MACHADO, D. P. N. O Impacto da Cultura sobre Ambiente Propício ao Desenvolvimento de Inovações. In: XXXVI Encontro da ANPAD, 2012, Rio de Janeiro. Anais do XXXVI Encontro da ANPAD, Rio de Janeiro, 2012.

SCHEIN, Edgar H. Coming to a new awareness of organizational culture. Sloan management review, v. 25, n. 2, p. 3-16, 1984.

SCHEIN, Edgar H. Organizational culture. American Psychological Association, 1990. 
What is culture? In: FROST, P. et al. (Org.). Reframing organizational culture. London: Sage, 1991.

SCHNEIDER, William E. Uma alternativa à reengenharia: um plano para fazer a cultura atual da sua empresa funcionar. Rio de Janeiro: Record, 1996.

SCHUMPETER, Joseph. O fenômeno fundamental do desenvolvimento econômico. A Teoria do

Desenvolvimento Econômico, 1985.

TAYLOR, James C.; BOWERS, David G. Survey of Organization. University of Michigan, 1972.

TIDD, J., et al. Managing innovation: integrating technological, market and organizational change. Chichester: John Wiley and Sons, 1997.

TROMPENAARS, Fons. Nas ondas da cultura: como entender a diversidade cultural nos negócios. São Paulo: Educator, 1994.

VAN DE VEN, A. H. Central problems in the management of innovation. Management Science, v. 32, n. 5, p. 590-607, maio de 1986.

VAN DE VEN, A. H; CHU, Y. A psychometric assessment of the Minnesota innovation survey Chapter 3. In: ; ANGLE, H.; POOLE, M. S.

(Org.). Research on the management of innovation: the Minnesota studies. New York: Oxford University, 1989.

VICENTI, Terezinha. Ambiente de inovação nas empresas de software de Blumenau, Santa Catarina. 2006, 201f. Dissertação (Mestrado em Administração) -Programa de Pós-Graduação em Administração, Universidade Regional de Blumenau, Blumenau, 2006.

ZIGER, B. J.; MAIDIQUE, M. A model of new product development: An empirical test. Management Science, v. 36, p. 867-883, 1990.

WHITTINGTON, Richard. Completing the practice turn in strategy research. Organization Studies, v. 27, n. 5, p. 613-634, 2006.

Data de recebimento: 28/09/2015

Data de aprovação: 13/12/2016

\section{SOBRE OS AUTORES}

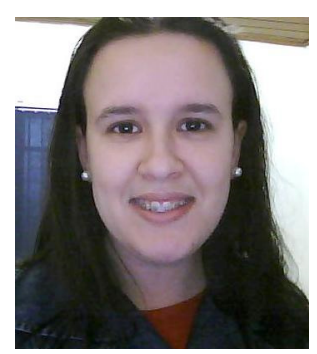

\section{Andressa Gonçalves}

Administradora graduada pela FAE Blumenau e mestra em Administração pela Universidade Regional de Blumenau (FURB). Pós-

Graduada em Planejamento e Gestão de Negócios. Atua como professora na faculdade IBES Sociesc nos cursos de Administração, Ciências Contábeis, Tecnólogos e Engenharias. Atuou como coordenadora dos cursos superiores de Recursos Humanos e Gestão Financeira da Faculdade Senac Blumenau. Atualmente é professora, pesquisadora e consultora empresarial.

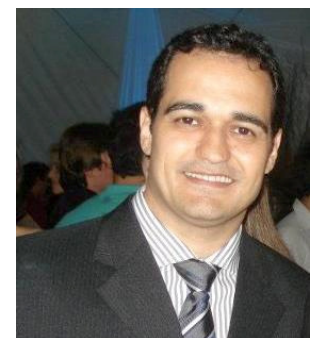

\section{Luciano de Castro} Carvalho

Possui graduação em Administração de Empresas pela Faculdade Estácio de Sá de Vila Velha (2004). Especialista em Controladoria e Finanças pela FUCAPE/ES, Mestre em Administração pela Universidade Regional de Blumenau - FURB e Doutor em Administração pela FGV/SP (com Bolsa FAPESP), com estagio em Indiana UniversityPardue Indianapolis University (IUPUI), Estados Unidos - com Bolsa CAPES -, com foco em Gestão deOperações e Competitividade.Membro doFórum de Inovação da EAESP/FGV, realiza pesquisas na área de Gestão de Operações, Qualidade em Serviços e Inovação. 


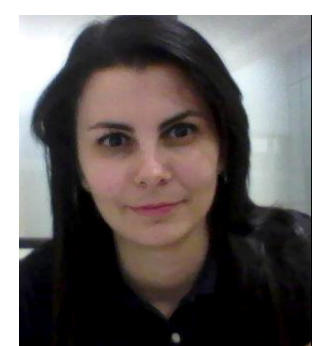

\section{Silvana Vieira Tambosi}

Mestre em Administração pela Universidade Regional de Blumenau (FURB). Pósgraduada em Administração com ênfase em gestão empresarial pela FAE Business School. Possui graduação em Administração pela Universidade do Sul de Santa Catarina (UNISUL) e graduação em Turismo pela Universidade Regional de Blumenau (FURB). Atualmente é professora da Universidade Regional de Blumenau e Designer Instrucional para modalidade EAD.

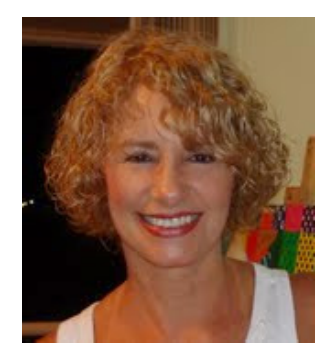

\section{Denise Del Pra Netto Machado}

Possui graduação em Administração de Empresas pela Faculdade Estácio de Sá de Vila Velha (2004). Especialista em Controladoria e Finanças pela FUCAPE/ES, Mestre em Administração pela Universidade Regional de Blumenau - FURB e Doutor em Administraçãopela FGV/SP (com Bolsa FAPESP), com estagio em Indiana UniversityPardue Indianapolis University (IUPUI), Estados Unidos-com Bolsa CAPES-, com foco em Gestão deOperações e Competitividade.Membro do Fórum de Inovação da EAESP/FGV, realiza pesquisas na área de Gestão de Operações, Qualidade em Serviços e Inovação.

\section{****}

